\title{
A Posterior Distribution for the Normal Mean Arising from a Ratio
}

\author{
Saralees Nadarajah ${ }^{1}$ and Arjun K. Gupta ${ }^{2}$ \\ ${ }^{1}$ University of Nebraska and ${ }^{2}$ Bowling Green State University
}

\begin{abstract}
It is shown that the most popular posterior distribution for the mean of the normal distribution is obtained by deriving the distribution of the ratio $X / Y$ when $X$ and $Y$ are normal and Student's $t$ random variables distributed independently of each other. Tabulations of the associated percentage points are given along with a computer program for generating them.
\end{abstract}

Key words: Normal distribution, posterior distribution for the normal mean, ratio of random variables, Student's $t$-distribution.

\section{Introduction}

The normal distribution is the most popular distribution in statistics. Suppose $x$ is an observation from a normal distribution with mean $\mu$ and precision $\lambda$ (precision $=1 /$ standard deviation). In a Bayesian context, one would usually have some prior knowledge about $\lambda$. For the past 40 to 50 years, the Student's $t$ distribution has been the most popular prior distribution because elicitation of prior information in various physical, engineering, and financial phenomena is closely associated with that distribution (see Kotz and Nadarajah (2004)). So, if we assume that

$$
\begin{aligned}
p(\mu, \lambda) & =p(\mu) p(\lambda) \\
& \propto\left(1+\frac{\lambda^{2}}{\nu}\right)^{-(1+\nu) / 2}
\end{aligned}
$$

(with the diffuse prior for $\mu$ ) then the posterior will be

$$
p(\mu, \lambda \mid x) \propto \lambda \exp \left\{-\frac{1}{2} \lambda^{2}(x-\mu)^{2}\right\}\left(1+\frac{\lambda^{2}}{\nu}\right)^{-(1+\nu) / 2} .
$$

Thus, the marginal posterior of $\mu$ will be

$$
p(\mu \mid x) \propto \int_{0}^{\infty} \lambda \exp \left\{-\frac{1}{2} \lambda^{2}(x-\mu)^{2}\right\}\left(1+\frac{\lambda^{2}}{\nu}\right)^{-(1+\nu) / 2} d \lambda .
$$


The density in (1.1) is the same as that of the ratio $X / Y$ when $X$ and $Y$ are normal and Student's $t$ random variables distributed independently of each other. Hence, calculating the marginal posterior of $\mu$ amounts to deriving the exact distribution of $X / Y$.

The distribution of $X / Y$ has been studied by several authors especially when $X$ and $Y$ are independent random variables and come from the same family. For instance, see Marsaglia (1965) and Korhonen and Narula (1989) for normal family, Press (1969) for Student's $t$ family, Basu and Lochner (1971) for Weibull family, Shcolnick (1985) for stable family, Hawkins and Han (1986) for non-central chi-squared family, Provost (1989) for gamma family, and Pham-Gia (2000) for beta family.

However, there is relatively little work of the above kind when $X$ and $Y$ belong to different families. In this note, we derive the marginal posterior distribution given by (1.1), which amounts to deriving the distribution of $|X / Y|$ when $X$ and $Y$ are independent random variables with the pdfs

$$
f_{X}(x)=\frac{1}{\sqrt{2 \pi} \sigma} \exp \left\{-\frac{x^{2}}{2 \sigma^{2}}\right\}
$$

and

$$
f_{Y}(y)=\frac{1}{\sqrt{\nu} B(\nu / 2,1 / 2)}\left(1+\frac{y^{2}}{\nu}\right)^{-(1+\nu) / 2},
$$

respectively, for $-\infty<x<\infty,-\infty<y<\infty, \sigma>0$ and $\nu>0$. We give explicit expressions for the pdf and the cdf of $|X / Y|$ (see Section 2). Tabulations of the percentage points associated with $|X / Y|$ are also provided (see Section 3) along with a computer program for generating them (see Appendix). The calculations of this note involve several special functions, including the complementary incomplete gamma function defined by

$$
\Gamma(a, x)=\int_{x}^{\infty} t^{a-1} \exp (-t) d t
$$

the Kummer function defined by

$$
\mathbf{K}(a, b ; x)=\frac{1}{\Gamma(a)} \int_{0}^{\infty} \exp (-x t) t^{a-1}(1+t)^{b-a-1} d t
$$

and the hypergeometric function defined by

$$
{ }_{2} F_{2}(a, b ; c, d ; x)=\sum_{k=0}^{\infty} \frac{(a)_{k}(b)_{k}}{(c)_{k}(d)_{k}} \frac{x^{k}}{k !}
$$


where $(e)_{k}=e(e+1) \cdots(e+k-1)$ denotes the ascending factorial. We also need the following important lemmas.

Lemma 1: (Equation (2.3.6.9), Prudnikov et al., 1986, volume 1) For $z>0$ and $p>0$,

$$
\int_{0}^{\infty} \frac{x^{\alpha-1} \exp (-p x)}{(x+z)^{\rho}} d x=\Gamma(\alpha) z^{\alpha-\rho} \mathbf{K}(\alpha, \alpha+1-\rho ; p z)
$$

Lemma 2: (Equation (2.8.3.5), Prudnikov et al., 1986, volume 2) For $z>0$, $\alpha>0$ and $c>0$,

$$
\begin{aligned}
& \int_{0}^{\infty} \frac{x^{\alpha-1}}{\left(x^{2}+z^{2}\right)^{\rho}} \Phi(-\sqrt{2} c x) d x \\
&= \frac{z^{\alpha-2 \rho}}{4} B\left(\frac{\alpha}{2}, \rho-\frac{\alpha}{2}\right)-\frac{c z^{1+\alpha-2 \rho}}{2 \sqrt{\pi}} B\left(\frac{\alpha+1}{2}, \rho-\frac{\alpha+1}{2}\right) \\
& \quad \times{ }_{2} F_{2}\left(\frac{\alpha+1}{2}, \frac{1}{2} ; \frac{3}{2}, \frac{\alpha+3}{2}-\rho ; c^{2} z^{2}\right) \\
& \quad-\frac{c^{2 \rho-\alpha}}{2 \sqrt{\pi}(2 \rho-\alpha)} \Gamma\left(\frac{\alpha+1}{2}-\rho\right) \\
& \quad \times{ }_{2} F_{2}\left(\rho, \rho-\frac{\alpha}{2} ; 1+\rho-\frac{\alpha}{2}, \frac{1-\alpha}{2}+\rho ; c^{2} z^{2}\right),
\end{aligned}
$$

where $\Phi(\cdot)$ denotes the cumulative distribution function of the standard normal distribution.

Further properties of the above special functions can be found in Prudnikov et al. (1986) and Gradshteyn and Ryzhik (2000).

\section{Exact Distribution of the Ratio}

Theorems 1 and 2 derive explicit expressions for the pdf and the cdf of | $X / Y \mid$ in terms of the Kummer, complementary gamma and the hypergeometric functions.

Theorem 1: Suppose $X$ and $Y$ are distributed according to (1.2) and (1.3), respectively. Then, the pdf of $Z=|X / Y|$ can be expressed as

$$
f_{Z}(z)=\frac{\sqrt{2 \nu}}{\sqrt{\pi} \sigma B(\nu / 2,1 / 2)} \mathbf{K}\left(1, \frac{3-\nu}{2}, 1 ; \frac{z^{2} \nu}{2 \sigma^{2}}\right),
$$

for $z>0$.

Proof: The general formula for the pdf of $|X / Y|$ is

$$
f_{Z}(z)=\int_{-\infty}^{\infty}|y|\left\{f_{X}(|y| z)+f_{X}(-|y| z)\right\} f_{Y}(y) d y .
$$


Since the given forms for $f_{X}(\cdot)$ and $f_{Y}(\cdot)$ are both symmetric around zero, the above can be expressed as

$$
\begin{aligned}
f_{Z}(z) & =4 \int_{0}^{\infty} y f_{X}(y z) f_{Y}(y) d y \\
& =\frac{4}{\sqrt{2 \pi \nu} \sigma B(\nu / 2,1 / 2)} \int_{0}^{\infty} y \exp \left(-\frac{y^{2} z^{2}}{2 \sigma^{2}}\right)\left(1+\frac{y^{2}}{\nu}\right)^{-(1+\nu) / 2} d y \\
& =\frac{\sqrt{2} \nu^{\nu / 2}}{\sqrt{\pi} \sigma B(\nu / 2,1 / 2)} \int_{0}^{\infty} \exp \left(-\frac{z^{2} w}{2 \sigma^{2}}\right)(w+\nu)^{-(1+\nu) / 2} d w,
\end{aligned}
$$

where the last step follows by substituting $w=y^{2}$. The result of the theorem follows by applying Lemma 1 to calculate the integral in (2.2).

Theorem 2: Suppose $X$ and $Y$ are distributed according to (1.2) and (1.3), respectively. Then, the cdf of $Z=|X / Y|$ can be expressed as

$$
\begin{aligned}
& F_{Z}(z)=\frac{4 z \sqrt{\nu}}{\sqrt{2 \pi} \sigma(\nu-1) B(\nu / 2,1 / 2)}{ }_{2} F_{2}\left(1, \frac{1}{2} ; \frac{3}{2}, \frac{3-\nu}{2} ; \frac{\nu z^{2}}{2 \sigma^{2}}\right) \\
& -\frac{(-1)^{3 \nu / 2} \Gamma((1-\nu) / 2)}{\sqrt{\pi} B(\nu / 2,1 / 2)}\left\{\Gamma\left(\frac{\nu}{2}\right)-\Gamma\left(\frac{\nu}{2},-\frac{\nu z^{2}}{2 \sigma^{2}}\right)\right\}
\end{aligned}
$$

for $z>0$.

Proof: The general formula for the cdf of $|X / Y|$ is

$$
F_{Z}(z)=\int_{-\infty}^{\infty}\left\{F_{X}(|y| z)-F_{X}(-|y| z)\right\} f_{Y}(y) d y
$$

Considering

$$
F_{X}(x)=\Phi\left(\frac{x}{\sigma}\right)
$$

(2.4) can be expressed as

$$
\begin{aligned}
F_{Z}(z) & =1-\frac{2}{\sqrt{\nu} B(\nu / 2,1 / 2)} \int_{-\infty}^{\infty} \Phi\left(-\frac{z|y|}{\sigma}\right)\left(1+\frac{y^{2}}{\nu}\right)^{-(1+\nu) / 2} d y \\
& =1-\frac{4 \nu^{\nu / 2}}{B(\nu / 2,1 / 2)} \int_{0}^{\infty} \Phi\left(-\frac{z y}{\sigma}\right)\left(y^{2}+\nu\right)^{-(1+\nu) / 2} d y
\end{aligned}
$$

where $\Phi(\cdot)$ denotes the cumulative distribution function of the standard normal distribution. By direct application of Lemma 2, the integral in (2.5) can be 
calculated as

$$
\begin{aligned}
& \int_{0}^{\infty} \Phi\left(\frac{z y}{\sigma}\right)\left(y^{2}+\nu\right)^{-(1+\nu) / 2} d y \\
= & \frac{B(1 / 2, \nu / 2)}{4 \nu^{\nu / 2}}-\frac{\nu^{(1-\nu) / 2} z}{(\nu-1) \sigma \sqrt{2 \pi}}{ }_{2} F_{2}\left(1, \frac{1}{2} ; \frac{3}{2}, \frac{3-\nu}{2} ; \frac{\nu z^{2}}{2 \sigma^{2}}\right) \\
& -\frac{z^{\nu} \Gamma((1-\nu) / 2)}{2 \sqrt{\pi} \nu(\sqrt{2} \sigma)^{\nu}}{ }_{2} F_{2}\left(\frac{1+\nu}{2}, \frac{\nu}{2} ; 1+\frac{\nu}{2}, \frac{1+\nu}{2} ; \frac{\nu z^{2}}{2 \sigma^{2}}\right) .
\end{aligned}
$$

The last term in (2.6) can be simplified by using the property that

$$
{ }_{2} F_{2}\left(\frac{1+\nu}{2}, \frac{\nu}{2} ; 1+\frac{\nu}{2}, \frac{1+\nu}{2} ; x\right)=\frac{\nu}{2}(-x)^{-\nu / 2}\left\{\Gamma\left(\frac{\nu}{2}\right)-\Gamma\left(\frac{\nu}{2},-x\right)\right\}
$$

The result of the theorem follows by combining (2.5)-(2.7).

Using special properties of the hypergeometric function, one can derive simpler forms for the distribution of $|X / Y|$ when $\nu$ takes integer values. This is illustrated in the corollary below.

Corollary 1: If $\nu=2,4,6,8,10$ then (2.3) reduces to

$$
\begin{gathered}
F_{Z}(z)=\exp (u)\{2 \Phi(\sqrt{2 u})-1\}+1-\exp (u) \\
F_{Z}(z)=\quad(1 / \sqrt{\pi})[-\sqrt{u}+\sqrt{\pi} \exp (u)\{2 \Phi(\sqrt{2 u})-1\} \\
\sqrt{\pi} u \exp (u)\{2 \Phi(\sqrt{2 u})-1\}-\sqrt{\pi}+\sqrt{\pi} \exp (u)-\sqrt{\pi} u \exp (u)] \\
F_{Z}(z)=\quad 1 /(4 \sqrt{\pi})\left[-5 \sqrt{u}+2 u^{3 / 2}-4 \sqrt{\pi} u \exp (u)\{2 \Phi(\sqrt{2 u})-1\}\right. \\
+4 \sqrt{\pi} \exp (u)\{2 \Phi(\sqrt{2 u})-1\}+2 \sqrt{\pi} u^{2} \exp (u)\{2 \Phi(\sqrt{2 u})-1\} \\
\left.+4 \sqrt{\pi}+4 \sqrt{\pi} u \exp (u)-4 \sqrt{\pi} \exp (u)-2 \sqrt{\pi} u^{2} \exp (u)\right] \\
F_{Z}(z)=\quad 1 /(24 \sqrt{\pi})\left[14 u^{3 / 2}-33 \sqrt{u}-4 u^{5 / 2}-24 \sqrt{\pi} u \exp (u)\{2 \Phi(\sqrt{2 u})-1\}\right. \\
+12 \sqrt{\pi} u^{2} \exp (u)\{2 \Phi(\sqrt{2 u})-1\}+24 \sqrt{\pi} \exp (u)\{2 \Phi(\sqrt{2 u})-1\} \\
4 \sqrt{\pi} u^{3} \exp (u)\{2 \Phi(\sqrt{2 u})-1\}-24 \sqrt{\pi}-24 \sqrt{\pi} u \exp (u) \\
\left.12 \sqrt{\pi} u^{2} \exp (u)+24 \sqrt{\pi} \exp (u)-4 \sqrt{\pi} u^{3} \exp (u)\right]
\end{gathered}
$$


and

$$
\begin{aligned}
F_{Z}(z)= & 1 /(192 \sqrt{\pi})\left[118 u^{3 / 2}-36 u^{5 / 2}-279 \sqrt{u}+8 u^{7 / 2}\right. \\
& +96 \sqrt{\pi} u^{2} \exp (u)\{2 \Phi(\sqrt{2 u})-1\}-192 \sqrt{\pi} u \exp (u)\{2 \Phi(\sqrt{2 u})-1\} \\
& 32 \sqrt{\pi} u^{3} \exp (u)\{2 \Phi(\sqrt{2 u})-1\}+192 \sqrt{\pi} \exp (u)\{2 \Phi(\sqrt{2 u})-1\} \\
& 8 \sqrt{\pi} u^{4} \exp (u)\{2 \Phi(\sqrt{2 u})-1\}+192 \sqrt{\pi}-96 \sqrt{\pi} u^{2} \exp (u) \\
& \left.192 \sqrt{\pi} u \exp (u)+32 \sqrt{\pi} u^{3} \exp (u)-192 \sqrt{\pi} \exp (u)-8 \sqrt{\pi} u^{4} \exp (u)\right],
\end{aligned}
$$

respectively, where $u=z^{2} /\left(2 \sigma^{2} \nu\right)$ and $\Phi(\cdot)$ denotes the cumulative distribution function of the standard normal distribution.

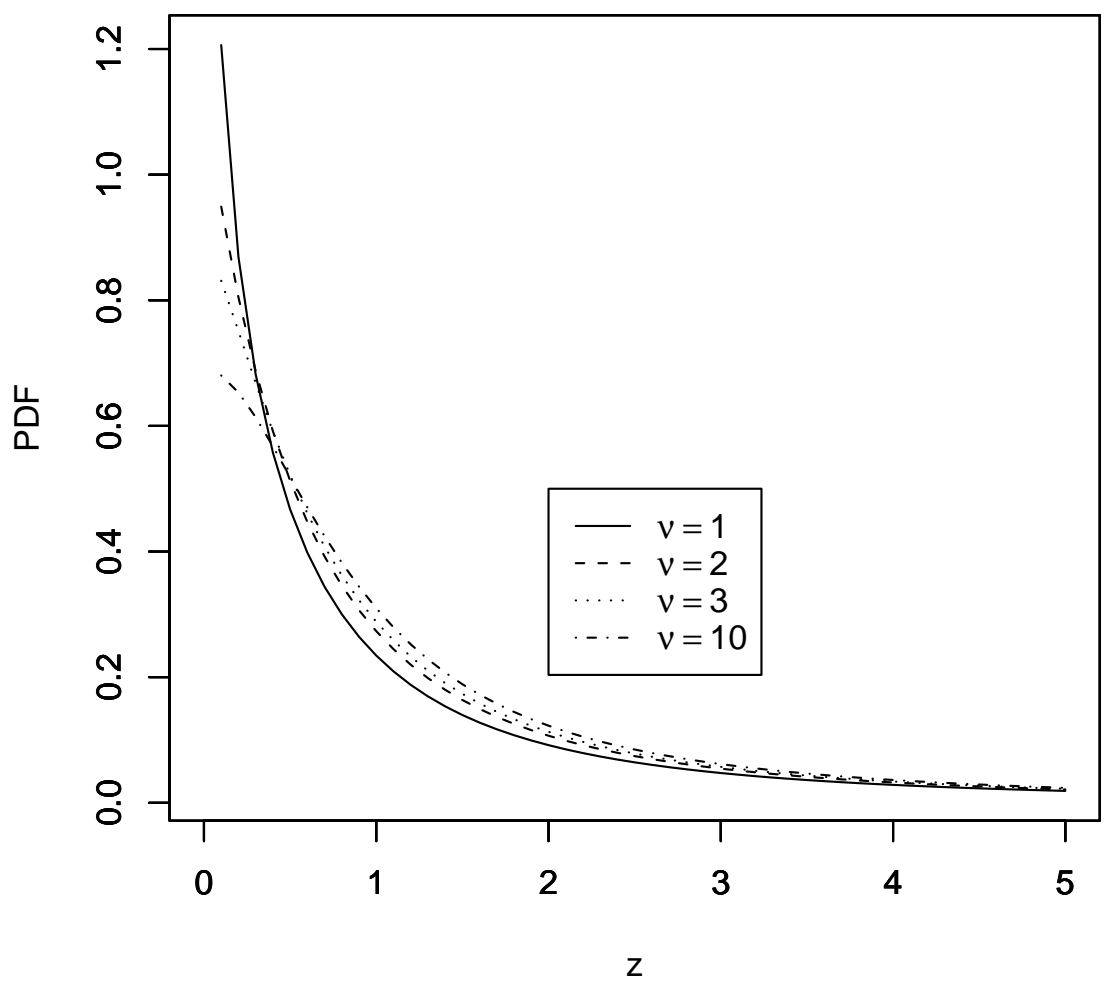

Figure 1: Plots of the pdf (2.1) for $\nu=1,2,3,10$ and $\sigma=1$ 
Table 1: Percentage points of $Z=|X / Y|$.

\begin{tabular}{ccccccc}
\hline$\nu$ & $p=0.01$ & $p=0.05$ & $p=0.1$ & $p=0.9$ & $p=0.95$ & $p=0.99$ \\
\hline 1 & 0.00285 & 0.01973 & 0.04805 & 4.95306 & 10.09401 & 50.78179 \\
2 & 0.00894 & 0.04613 & 0.09629 & 5.55459 & 11.23967 & 56.41010 \\
3 & 0.01137 & 0.05731 & 0.11686 & 5.79018 & 11.69280 & 58.64533 \\
4 & 0.01254 & 0.06297 & 0.12751 & 5.91491 & 11.93352 & 59.83438 \\
5 & 0.01321 & 0.06628 & 0.13389 & 5.99189 & 12.08233 & 60.56984 \\
6 & 0.01365 & 0.06844 & 0.13811 & 6.04406 & 12.18327 & 61.06894 \\
7 & 0.01395 & 0.06996 & 0.14109 & 6.08172 & 12.25617 & 61.42955 \\
8 & 0.01418 & 0.07109 & 0.14331 & 6.11017 & 12.31128 & 61.70216 \\
9 & 0.01436 & 0.07196 & 0.14503 & 6.13242 & 12.35439 & 61.91543 \\
10 & 0.01450 & 0.07266 & 0.14640 & 6.15029 & 12.38903 & 62.08681 \\
11 & 0.01461 & 0.07322 & 0.14751 & 6.16496 & 12.41747 & 62.22752 \\
12 & 0.01470 & 0.07369 & 0.14844 & 6.17721 & 12.44123 & 62.34511 \\
13 & 0.01478 & 0.07408 & 0.14922 & 6.18760 & 12.46138 & 62.44483 \\
14 & 0.01485 & 0.07442 & 0.14988 & 6.19651 & 12.47869 & 62.53048 \\
15 & 0.01491 & 0.07471 & 0.15046 & 6.20426 & 12.49371 & 62.60483 \\
16 & 0.01496 & 0.07496 & 0.15096 & 6.21104 & 12.50687 & 62.66997 \\
17 & 0.01500 & 0.07519 & 0.15140 & 6.21703 & 12.51850 & 62.72752 \\
18 & 0.01504 & 0.07539 & 0.15180 & 6.22236 & 12.52885 & 62.77874 \\
19 & 0.01508 & 0.07556 & 0.15215 & 6.22714 & 12.53812 & 62.82460 \\
20 & 0.01511 & 0.07572 & 0.15246 & 6.23144 & 12.54646 & 62.86591 \\
\hline
\end{tabular}

Figure 1 illustrates possible shapes of (2.1) for a range of values of $\nu$. Note that the shapes are unimodal and that the value of $\nu$ largely dictates the behavior of the pdf near $z=0$.

\section{Percentiles}

In this section, we provide tabulations of percentage points $z_{p}$ associated with the cdf (2.3). These values are obtained numerically by solving the equation

$$
\begin{aligned}
& \frac{4 z_{p} \sqrt{\nu}}{\sqrt{2 \pi} \sigma(\nu-1) B(\nu / 2,1 / 2)}{ }_{2} F_{2}\left(1, \frac{1}{2} ; \frac{3}{2}, \frac{3-\nu}{2} ; \frac{\nu z_{p}^{2}}{2 \sigma^{2}}\right) \\
& -\frac{(-1)^{3 \nu / 2} \Gamma((1-\nu) / 2)}{\sqrt{\pi} B(\nu / 2,1 / 2)}\left\{\Gamma\left(\frac{\nu}{2}\right)-\Gamma\left(\frac{\nu}{2},-\frac{\nu z_{p}^{2}}{2 \sigma^{2}}\right)\right\}=p .
\end{aligned}
$$

Evidently, this involves computation of the hypergeometric and the incomplete gamma functions and routines for this are widely available. We used the functions 
hypergeom $([\cdot, \cdot],[\cdot, \cdot], \cdot)$ and GAMMA $(\cdot, \cdot)$ in the algebraic manipulation package, MAPLE. Table 1 below provides the numerical values of $z_{p}$ for $\nu=1,2, \ldots, 100$ and $p=0.01,0.05,0.1,0.9,0.95,0.99$. It is assumed, without loss of generality, that $\sigma=1$. A longer version of the table can be found in the electronic version of this paper.

It is expected that this table will be of use to many just like the tables for the normal and $t$ distributions are. Similar tabulations could be easily derived for other values of $\nu$ and $p$ by using the hypergeom $([\cdot, \cdot],[\cdot, \cdot], \cdot)$ and GAMMA $(\cdot, \cdot)$ functions in MAPLE. A sample program is shown in the Appendix below.

\section{Acknowledgments}

The authors would like to thank the Editor and the referee for carefully reading the paper and for their help in improving the paper.

\section{Appendix}

The following program in MAPLE can be used to generate tables similar to that presented in Section 3.

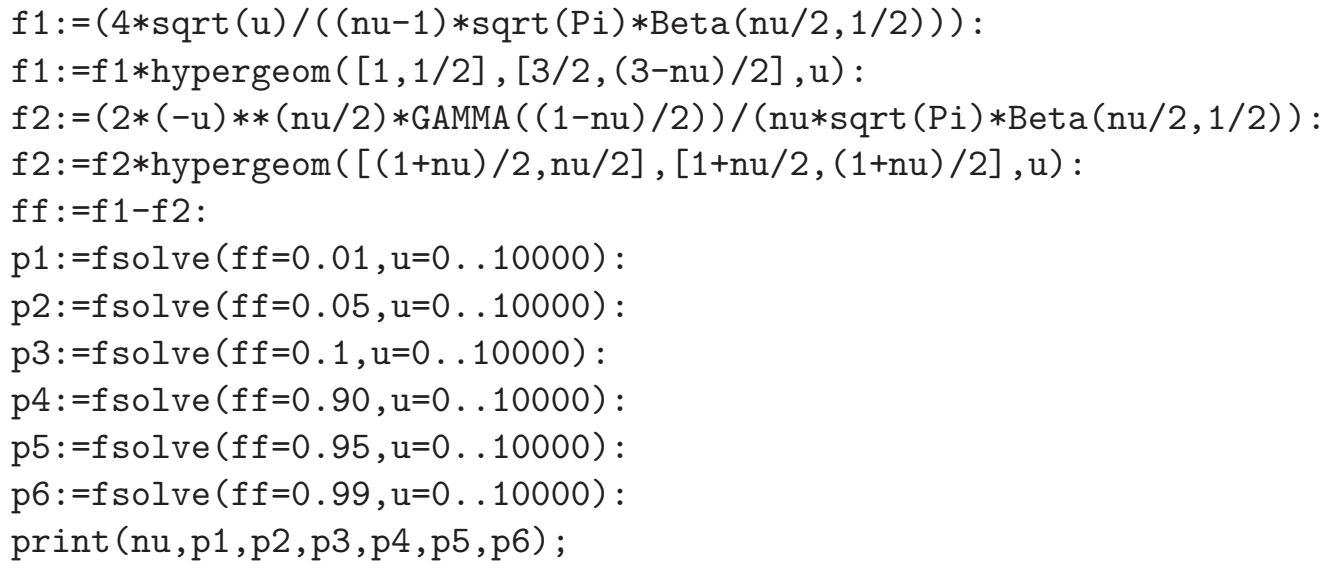

\section{References}

Basu, A. P. and Lochner, R. H. (1971). On the distribution of the ratio of two random variables having generalized life distributions. Technometrics 13, 281-287.

Gradshteyn, I. S. and Ryzhik, I. M. (2000). Table of Integrals, Series, and Products (sixth edition). Academic Press, San Diego. 
Hawkins, D. L. and Han, C.-P (1986). Bivariate distributions of some ratios of independent noncentral chi-square random variables. Communications in StatisticsTheory and Methods 15, 261-277.

Korhonen, P. J. and Narula, S. C. (1989). The probability distribution of the ratio of the absolute values of two normal variables. Journal of Statistical Computation and Simulation 33, 173-182.

Kotz, S. and Nadarajah, S. (2004). Multivariatet Distributions and Their Applications. Cambridge University Press, New York.

Marsaglia, G. (1965). Ratios of normal variables and ratios of sums of uniform variables. Journal of the American Statistical Association 60, 193-204.

Pham-Gia, T. (2000). Distributions of the ratios of independent beta variables and applications. Communications in Statistics - Theory and Methods 29, 2693-2715.

Press, S. J. (1969). The $t$ ratio distribution. Journal of the American Statistical Association 64, 242-252.

Provost, S. B. (1989). On the distribution of the ratio of powers of sums of gamma random variables. Pakistan Journal Statistics 5, 157-174.

Prudnikov, A. P., Brychkov, Y. A. and Marichev, O. I. (1986). Integrals and Series (volumes 1, 2 and 3). Gordon and Breach Science Publishers, Amsterdam.

Shcolnick, S. M. (1985). On the ratio of independent stable random variables. Stability Problems for Stochastic Models (Uzhgorod, 1984), 349-354, Lecture Notes in Mathematics, 1155, Springer.

Received January 17, 2006; accepted February 26, 2006.

Saralees Nadarajah

School of Mathematics

University of Manchester

Manchester M60 1QD, UK

saralees.nadarajah@manchaster.ac.uk

Arjun K. Gupta

Department of Mathematics and Statistics

Bowling Green State University

Bowling Green, Ohio 43403

gupta@bgsu.edu 